\title{
EDITOR'S NOTE: VOLUME OVERVIEW
}

With the advent of increasingly accessible Internetbased technologies, it is not difficult to envision a future wherein the practice of telerehabilitation is the norm, rather than an innovative exception.

This issue of the International Journal of Telerehabilitation (IJT) focuses on three powerful delivery systems: Voice over the Internet Protocol (VolP); YouTube; and e-mail.

Clinicians are increasingly employing Voice over the Internet Protocol (VoIP) systems such as Skype, Adobe ConnectNow, and ooVoo to conduct telepractice sessions. This is not surprising, as VolP systems can offer inexpensive and easy ways to deliver telerehabilitation (TR) services. But how might a telerehabilitation clinician determine if their VoIP system is truly secure and private? Valerie Watzlaf, a Registered Health Information Administrator (RHIA), and colleagues Sohrab Moeini and Patti Firouzan address this question. They present HIPAAbased criteria for both therapists and healthcare facilities to consider, and conclude that "therapists must weigh the risks and benefits when deciding to use VolP software for TR."

Another excellent contribution to this issue concerns the application of "new media." M. Hunter Manasco, Nicholas Barone, and Amanda Brown describe the potential role of YouTube in telerehabilitation. Their innovative efforts suggest possibilities that therapists from all rehabilitation disciplines might consider.

Min Jung Kim, Julie A.G. Stierwalt, and Leonard L. LaPointe consider the use of email intervention following traumatic brain injury. These authors' insightful and detailed review of the literature, and elegant, evidencebased case reports are not to be missed!

And finally, IJT is honored to publish a White Paper that was generated by the American Telemedicine Association's Telerehabilitation Special Interest Group: "A Blueprint for Telerehabilitation Guidelines."

\section{CALL FOR SUBMISSIONS}

The next volume of the International Journal of Telerehabilitation will be published in spring, 2011. We cordially invite your submissions by February 15, 2011, and accept original research, case studies, viewpoints, technology reviews, book reviews, and country reports that detail the current status of telerehabilitation. We also welcome new peer-reviewers and are open to guest editors with ideas for special, thematically focused issues. Please be in touch if you are interested!

\section{ACKNOWLEDGMENTS}

This issue of the International Journal of Telerehabilitation would not be possible without the support of dedicated authors, reviewers, colleagues at the Rehabilitation Research Engineering Center on Telerehabilitation (especially Joseph Ruffing, graphic designer, and Deborah Keelan, proof-reader), and our publisher, Timothy S. Deliyannides, Head, Department of Information Systems, University Library System, and Vanessa Gabler, Electronic Publications Associate at the University of Pittsburgh.

Many thanks to all!

Ellen R. Cohn, PhD

Editor 
